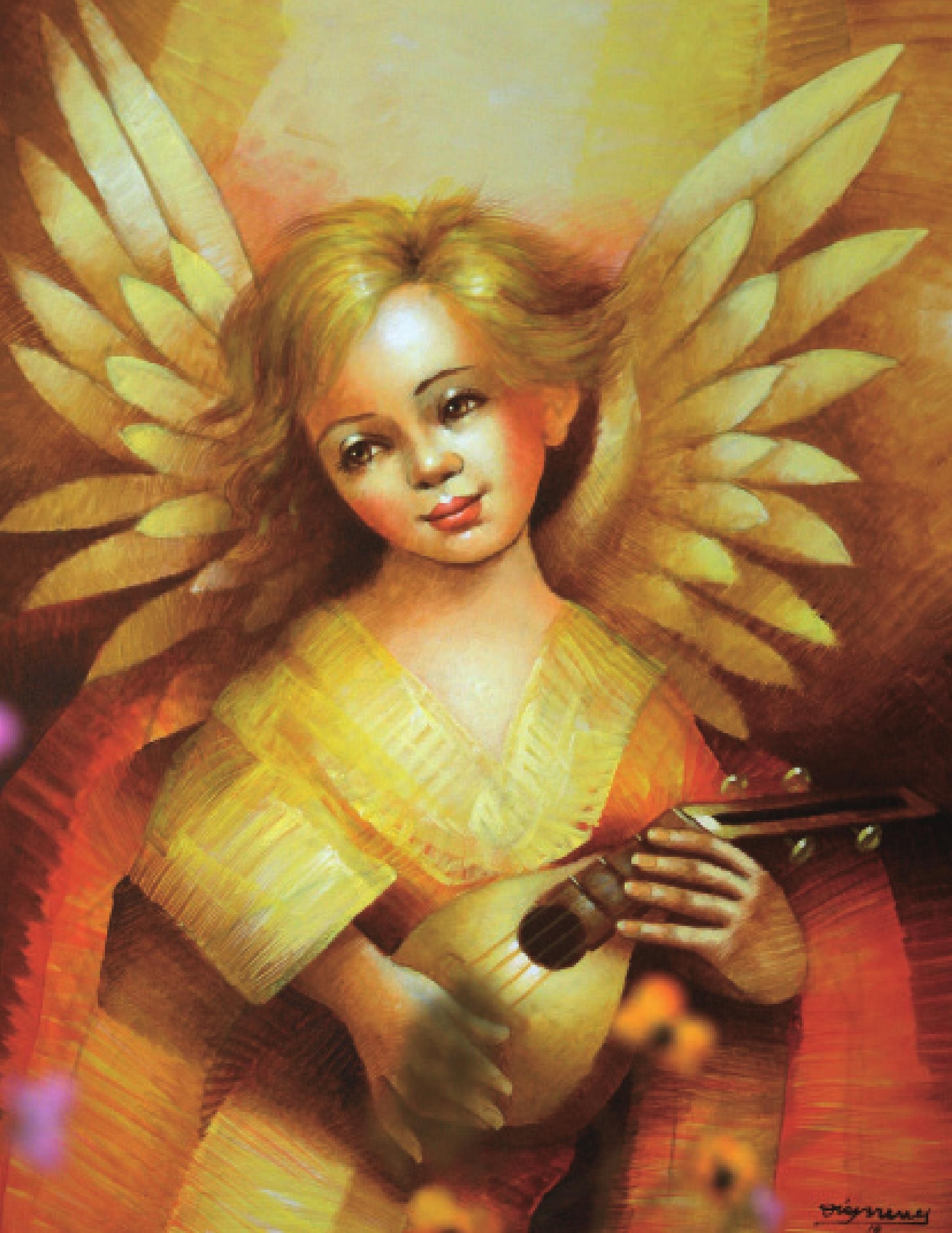




\title{
5 Mortalidad infantil en el año 2009 y determinantes sociales
}

\author{
Ma. Guadalupe Romero-A. * Ingrid Carolina Garay R. **
}

RESUMEN. Las condiciones sociales, económicas, políticas y ambientales, y en este caso, territoriales en las cuales la gente nace, vive y envejece, son aspectos claves en la salud de la población. La mortalidad infantil, es un indicador relevante del nivel de desarrollo de un país. Las mejoras en la salud sólo podrán suceder si se focaliza en los determinantes sociales de la salud. El presente trabajo de investigación revisa los determinantes de la mortalidad infantil a saber: territorialidad, definido por las regiones departamentales; índice de educación e índice de ingreso. Esta investigación es de diseño descriptivo retrospectivo. Los datos de mortalidad infantil se tomaron del Boletín Epidemiológico de la Secretaría de Salud correspondiente al año 2009. Los índices de educación y de ingreso se obtuvieron del documento del Programa de las Naciones Unidas para el Desarrollo (PNUD) denominado: Desarrollo Humano en Honduras: limitado por la inequidad territorial del año 2009. Los departamentos con mayores tasas de mortalidad infantil para el 2009 en su orden fueron: Francisco Morazán, Cortés, Copán, Intibucá, Lempira y Ocotepeque. La velocidad de muerte fue de 43 por semana epidemiológica. Los índices de educación y de ingreso más altos, corresponden a los departamentos de Francisco Morazán, Cortés, Atlántida, por lo que se concluye que aun cuando los departamentos mencionados tienen mayores ventajas son los que presentan mayores tasas de mortalidad, haciendo hincapié en que se debe realizar un análisis más exhaustivo de los determinantes que permitan explicar la mortalidad infantil.

Palabras claves: mortalidad infantil, territorialidad, índice de educación, índice de ingreso.

ABSTRACT. The social, economic, political and environmental conditions, in this case, land in which people are born, live and age, are keys to the health of the population. Infant mortality is an important indicator of the level of development of a country. Improvements in population health can only happen if you focus on the social determinants of health. This research paper reviews the determinants of infant mortality namely territoriality, defined by regions departmental education index and income index. This research is retrospective design. Infant mortality data were taken of the Epidemiological Bulletin of the Ministry of Health for the year 2009. The index of education and income, were taken from the UNDP document called Human Development in Honduras: limited by territorial inequality in 2009. The data show that the departments with the highest rates of infant mortality, in order: Francisco Morazán, Cortes, Copan, Intibucá, Lempira and Ocotepeque. The death rate is 43 epidemiological week for that year. The rates of education and income, the higher are the departments of Francisco Morazán, Cortés, Atlántida, so we conclude that even if these departments, seem to have greater advantages, are those with higher mortality rates, making should be emphasized that most cursory analysis of the determinants that explain child mortality.

Keywords: infant mortality, territoriality, education index, income index.

\section{Introducción}

La mortalidad infantil es uno de los problemas estudiados a nivel mundial, sobretodo porque se estima que millones de niños/as mueren por causas evitables. Conocer sus causas directas y sus determinantes es lo que permite monitorear el efecto de los programas y de sus diferentes intervenciones en la solución del problema (Delgado, 2003).

En los países en vías de desarrollo, se logró plasmar con mayor precisión la necesidad de revisar otro tipo de determinantes, que no fueran la prevención de ciertas enfermedades, que también tenían presencia en la mortalidad y en este caso, la mortalidad infantil.

La Cumbre Mundial a favor de la Infancia fijó para el año 2000 metas precisas, lo que requería evaluaciones que permitieran visualizar los avances. La información fue necesaria para que cada país asignará recursos y con esto, obtener resultados con eficiencia (Ahmad, López, \& Inoue, 2000). Uno de los logros más importantes provocados por esta cumbre, fue la mejoría de los sistemas de recolección de información y procesamiento de datos

Recibido: julio 2013 / Aceptado y versión final agosto 2013.

* Msc. en Salud Pública, Msc. en Administración de Servicios de Salud, Doctorante en Ciencias con Orientación en Ciencias de la Administración. mgromeroa@yahoo.com

** Ingeniera en Ciencias Ambientales. Máster en Ciencias, Tecnología y Gestión del Agua. PhD. e-mail: Ingrid icgaray@usal.es 
sobre la situación de la infancia. Los datos disponibles evidenciaron el aumento o la disminución de las tasas de mortalidad infantil en regiones y países.

El presente trabajo realiza una revisión documental de los datos de mortalidad infantil, que aparecen en el Boletín Epidemiológico del año 2009 de la Secretaría de Salud, y los relaciona con los datos que el PNUD presenta en dos de los componentes del Índice de Desarrollo Humano: el índice de educación ${ }^{1}$ y el índice de ingreso ${ }^{2}$, por región departamental de ese mismo año.

\section{Metodología}

Esta investigación responde a un diseño descriptivo y retrospectivo. Se revisaron los datos proporcionados por el Boletín Epidemiológico de la Dirección de Vigilancia de la Salud del año 2009, con respecto a mortalidad infantil por departamentos y los datos proporcionados en el Informe de Desarrollo Humano del PNUD del año 2009, con respecto a la inequidad territorial, así como también los datos suministrados por los componentes del índice de educación y del índice de ingreso por departamento, que aparecen como un todo en el Informe del Índice de Desarrollo Humano del año 2009 (Salud, 2009 y PNUD, 2009).

Las variables analizadas fueron: mortalidad infantil por región departamental, índice de educación e índice de ingreso.

\section{Aspectos conceptuales}

La mortalidad infantil es una variable demográfica que se define como la muerte de un niño(a) en la edad comprendida entre los 0 y 11 meses y 29 dias (Honduras, 2008). Es un indicador relevante del nivel de desarrollo social de un país, razón por la cual ha sido considerado entre los Objetivos de Desarrollo del Milenio (ODM) de la Organización de las Naciones Unidas (ONU), especí- ficamente el ODM4: Reducir la mortalidad de los niños las menores de 5 años (PNUD, 2008).

Aun cuando la reducción de la mortalidad en la niñez no haya sido tan acelerada como lo requiere el cumplimiento de las metas al 2015, se ha dado una reducción importante en el país. Hay avances en el proceso de la atención primaria de la salud, pero los mayores logros se han visto obstaculizados por la dimensión de otras limitantes y desafíos que hay que enfrentar. Existen factores de naturaleza estructural que deben ser considerados para comprender las dificultades que enfrenta el cumplimiento de las metas de este Objetivo de Desarrollo del Milenio. La situación económica del hogar es un factor clave en la explicación de las tasas de mortalidad en la niñez. El nivel de educación de la madre, también es clave, ya que los niños y niñas de madres sin educación tienen casi tres veces mayor riesgo de morir que el grupo de niños y niñas cuyas madres tienen educación secundaria y superior. El área de residencia también es relevante, en vista de que los niños y niñas que viven en el área rural tienen una y media más veces más riesgo de morir que el mismo grupo de edad que vive en el área urbana (PNUD, 2008).

La Organización Mundial de la Salud (OMS) ha hecho énfasis en los determinantes sociales de la salud, de tal forma, que a partir del año 2005, crea la Comisión de Determinantes Sociales de la Salud (CDSS), con el fin de aportar conocimientos y sensibilizar sobre el tema. Los ejes de la desigualdad están relacionados con el nivel socioeconómico y pueden expresarse como bajo nivel de ingresos, nivel de instrucción y lugar de residencia (Dirección de Planificación, 2009).

La CDSS tiene una visión global de los determinantes sociales de la salud y de las grandes desigualdades que repercuten en las condiciones de vida de la población de forma inmediata y visible. Entre las determinantes se encuentran, el desarrollo económico y la educación. Tradicionalmente, la sociedad espera que el sector sanitario

1. Medida por la tasa de alfabetización de adultos y la tasa bruta combinada de matriculación en educación primaria, secundaria y superior, así como los años de duración de la educación obligatoria.

2. Calculado a partir del ingreso percapita 
se ocupe de aspectos específicos que afectan a la salud y a la enfermedad, pero la elevada carga de morbilidad causante de mortalidad prematura se debe a las condiciones en que las personas nacen, crecen, viven, trabajan (OMS C. d., 2009).

Las cifras sobre alfabetismo, son un crudo reflejo del acceso a la educación, tan necesaria para llevar una vida productiva en la sociedad actual. Aprender a leer y escribir es el primer paso de una persona hacia el aprendizaje y la adquisición de conocimientos de tal forma que las cifras sobre alfabetismo son esenciales en cualquier medición del desarrollo humano.

Otro componente importante, es el manejo de los recursos que se requieren para una vida decente. El indicador de ingreso del que se dispone, es el ingreso per cápita, que tiene cobertura más amplia (PNUD, 1990).

En 1979, Hugo Behm propuso el marco conceptual histórico-estructural para el abordaje de la mortalidad infantil y afirmó que la relación entre muertes con condiciones socioeconómicas, es bien conocida, aunque el modo de acción y el peso relativo de los diversos factores intervinientes, no es un asunto muy bien dilucidado. En el análisis de ese marco, Behm asoció al estudio de la mortalidad infantil con distintas variables como: nivel de ingreso, nivel de educación, grupos étnicos y clases sociales. Los análisis de estructuras arriban a generalidades obvias que coinciden en el punto que son los pobres los que más se mueren (Behm, 2011).

El PNUD establece que una de las grandes barreras al desarrollo humano de las personas en países como Honduras, derivan de las inequidades de carácter territorial. Estas inequidades son adscriptivas y van asociadas al nacer o vivir en un territorio determinado, lo que implica ventajas o desventajas sociales para que las personas puedan desarrollar sus potencialidades, por lo que se puede mencionar que hay regiones o territorios que generan ventajas sociales a sus habitantes debido al relativo acceso a servicios públicos o privados. En cambio, hay otros, como señala la CEPAL, que..."perpetúan y ahondan el rezago económico y social”... (PNUD, 2009). Estableciendo el concepto, se designa como inequidad territorial a las desigualdades geográficas que se consideran injustas y socialmente remediables.

El informe establece que existen grandes brechas en las dimensiones básicas entre regiones o territorios de Honduras y al desagregar los índices de educación y de ingreso por regiones y área geográfica, se reflejan las condiciones de inequidad económica y social en que se encuentran las personas que viven en determinados territorios cuyos niveles de ingreso, de acceso a la salud y a la educación difieren con respecto a otros territorios del país (PNUD, 2009).

El PNUD en el año 2009 realizó la creación del índice de educación, considerando aspectos de cobertura y no tanto de calidad educativa. Para realizarlo, se tomó como base las pruebas de rendimiento académico aplicadas por la Unidad Externa de Medición de la Calidad de la Educación (UMCE) a los/as alumnos/as de nivel primario y las pruebas de aptitud académica aplicadas por la Universidad Nacional Autónoma de Honduras (UNAH) a los alumnos/as de nivel secundario que aspiran a ingresar a la misma. La UMCE, adopta una definición que establece que el rendimiento, aptitud o desempeño académico, muestra el nivel de conocimiento demostrado en un área o materia comparada con la norma de edad y nivel académico. Lo anterior depende de varios factores, entre los que se encuentran el estado nutricional de los/ las estudiantes, el ambiente en el que se desenvuelven, su edad y grado escolar (PNUD, 2009, FEREMA, 2010). A nivel del ingreso, el ingreso per cápita, se encuentra afectado por el tipo de actividad económica en que están insertas las personas en el mercado laboral, ya que unas son más productivas que otras y eso influye directamente en la remuneración (PNUD, 2009).

\section{Resultados de investigación}

En el período de enero-diciembre de 2009 (semana epidemiológica 52), se notificaron 2,212 muertes de niños(as) menores de 1 año. La velocidad de muerte fue de 43 muertes infantiles por semana epidemiológica. Los departamentos que registraron mayor velocidad de 
muerte fueron: Francisco Morazán, con una velocidad por semana epidemiológica de 13 muertes infantiles; Cortés, con una velocidad de muerte por semana epidemiológica de 9 muertes infantiles, en tercer lugar se encontró el departamento de Copán, con 3 muertes por semana epidemiológica. A nivel porcentual, estas regiones departamentales también registraron los porcentajes más altos: Francisco Morazán, Cortés y Copán. El rango se encuentra entre 8 (valor mínimo) y 684 (valor máximo), (Cuadro 1).

Cuadro 1. Casos notificados de mortalidad infantil por departamento, año 2009.

\begin{tabular}{|c|c|c|}
\hline DEPARTAMENTO & $\begin{array}{c}\text { CASOS } \\
\text { NOTIFICADOS }\end{array}$ & PORCENTAJES \\
\hline Atlántida & 83 & 3.75 \\
\hline Colón & 48 & 2.16 \\
\hline Comayagua & 54 & 2.44 \\
\hline Copán & 163 & 7.36 \\
\hline Cortés & 483 & 21.83 \\
\hline Choluteca & 116 & 5.23 \\
\hline El Paraíso & 51 & 2.30 \\
\hline Francisco Morazán & 684 & 30.92 \\
\hline Gracias a Dios & 20 & 0.90 \\
\hline Intibucá & 85 & 3.84 \\
\hline Islas de la bahía & 8 & 0.36 \\
\hline La Paz & 49 & 2.21 \\
\hline Lempira & 108 & 4.88 \\
\hline Ocotepeque & 37 & 1.67 \\
\hline Olancho & 109 & 4.92 \\
\hline Santa Bárbara & 35 & 1.58 \\
\hline Valle & 12 & 0.54 \\
\hline Yoro & 67 & 3.02 \\
\hline Total & 2,212 & 100.00 \\
\hline
\end{tabular}

Fuente: Boletín Epidemiológico. República de Honduras. 2009.

La tasa más alta de mortalidad infantil se registró en el departamento de Francisco Morazán (30.92\%), seguido de Cortés (21.83\%); el tercer lugar lo ocupó Copán (7.36\%); el cuarto lugar, Choluteca (5.23\%); el quinto lugar, Olancho (4.92\%); el sexto lugar, Lempira (4.88\%) y el séptimo lugar, Yoro (3.02\%), (Gráfico 1).

Los departamentos que presentaron los mayores porcentajes de mortalidad infantil y las mayores tasas,
Gráfico 1. Tasa de Mortalidad Infantil, por departamento 2009

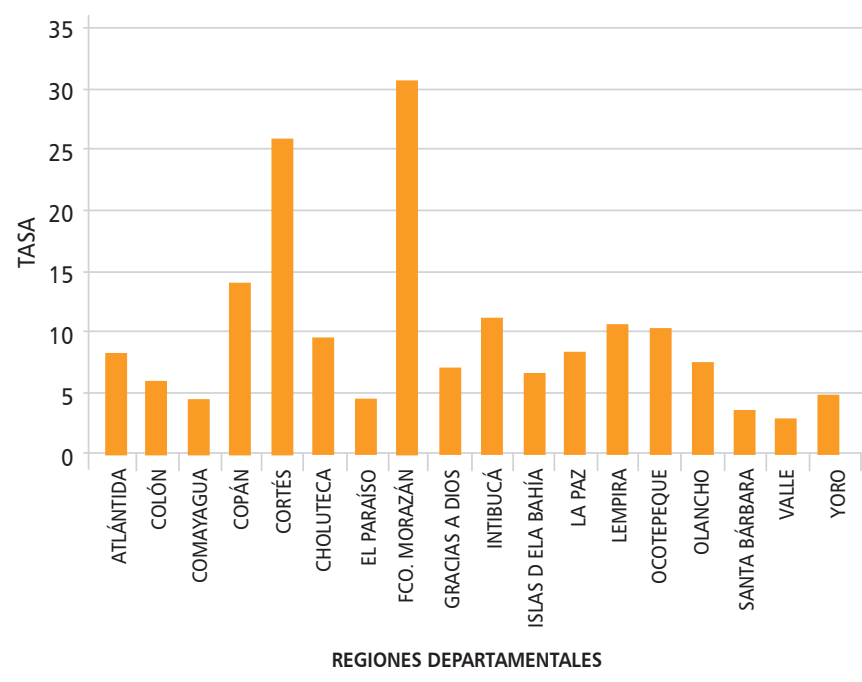

Fuente: Boletín Epidemiológico. República de Honduras. 2009

están relacionados con los que cuentan con hospitales nacionales de referencia a los que se les envía los casos, que por diferentes razones no pueden ser atendidos en el resto de los establecimientos de la Red Hospitalaria. Estos hospitales tienen en su haber recurso humano con altas capacidades como sub especialidades y por ende, mejor equipados. Algunos ejemplos de esto son el Hospital Escuela y el Hospital Mario Catarino Rivas. La gravedad de los casos que son referidos marca la pauta de este comportamiento. En cuanto a Copán, la relación con los índices de educación y de ingreso que se destaca en el documento del PNUD señala que los valores se encuentran por debajo de la media tanto a nivel del índice de educación, como del índice de ingreso.

En ese sentido, estudios realizados en Chile en el año 2010 en el que interaccionaban variables asociadas, v.g, mortalidad infantil y nivel educativo del padre y de la madre, y donde se realizaron nueve combinaciones de años de estudios aprobados (AEA) por ambos, muestran que existe una interacción aditiva de tipo sinérgico entre los años de estudio aprobados por ambos progenitores y la tasa de mortalidad infantil, siendo un riesgo atribuible mayor a la mortalidad infantil, la coexistencia de un nivel educativo bajo en ambos padres (Medina \& Cerda, 2010). 
En la misma condición se encuentran Choluteca, Olancho y Lempira que ocupan el último lugar en relación al índice de educación. En cuanto a Yoro, los datos reflejan que los índices de educación y el de ingresos se encuentran superiores a la media, razón por la cual se deberá realizar la relación con otras variables que abordarían los motivos por los cuales ocupa el séptimo lugar en esta casuística. En este caso, es importante destacar que desde el punto de acceso a la atención hospitalaria, este departamento cuenta con tres hospitales a saber: Subirana en Yoro, la cabecera departamental; otro en la ciudad de El Progreso y otro en Olanchito, todos con atención materno-infantil.

\section{Cuadro 2. Valores de índice de educación por departa- mento, 2009.}

\begin{tabular}{lc} 
& DEPARTAMENTO \\
\hline Atlántida & .774 \\
Colón & .711 \\
Comayagua & .720 \\
\hline Copan & .625 \\
\hline Cortés & .787 \\
\hline Choluteca & .683 \\
\hline El Paraíso & .676 \\
\hline Francisco Morazán & .805 \\
\hline Gracias a Dios & .698 \\
Intibucá & .672 \\
\hline Islas de la Bahía & .839 \\
\hline La Paz & .716 \\
Lempira & .605 \\
\hline Ocotepeque & .646 \\
Olancho & .658 \\
Santa Barbara & .618 \\
Valle & .693 \\
Yoro & .714 \\
\hline
\end{tabular}

Fuente: Desarrollo Humano en Honduras limitado por la Inequidad. 2009. PNUD.
Los datos de los departamentos de Francisco Morazán y Cortés, incluyen a la Región Metropolitana de Tegucigalpa y la Región Metropolitana de San Pedro Sula, respectivamente.

El Cuadro 2 muestra que el índice de educación ${ }^{3}$ más alto, se encuentra en el departamento de Francisco Morazán, seguido a continuación por Cortés, en tercer lugar, Atlántida y en cuarto lugar, Comayagua. El rango se encuentra entre 0.605 (valor mínimo) y 0.805 (valor máximo), (Gráfico 2).

A nivel de ingreso ${ }^{4}$, el Cuadro 3 muestra que el primer lugar lo ocupa el departamento de Francisco Morazán; el segundo lugar, el departamento de Cortés; el tercer lugar, el departamento de Islas de la Bahía y el cuarto lugar, el departamento de Atlántida. El rango se encuentra entre .453 (valor mínimo) y .682 (valor máximo), (Gráfico 3).

\section{Gráfico 2. Índice de Educación, 2009}

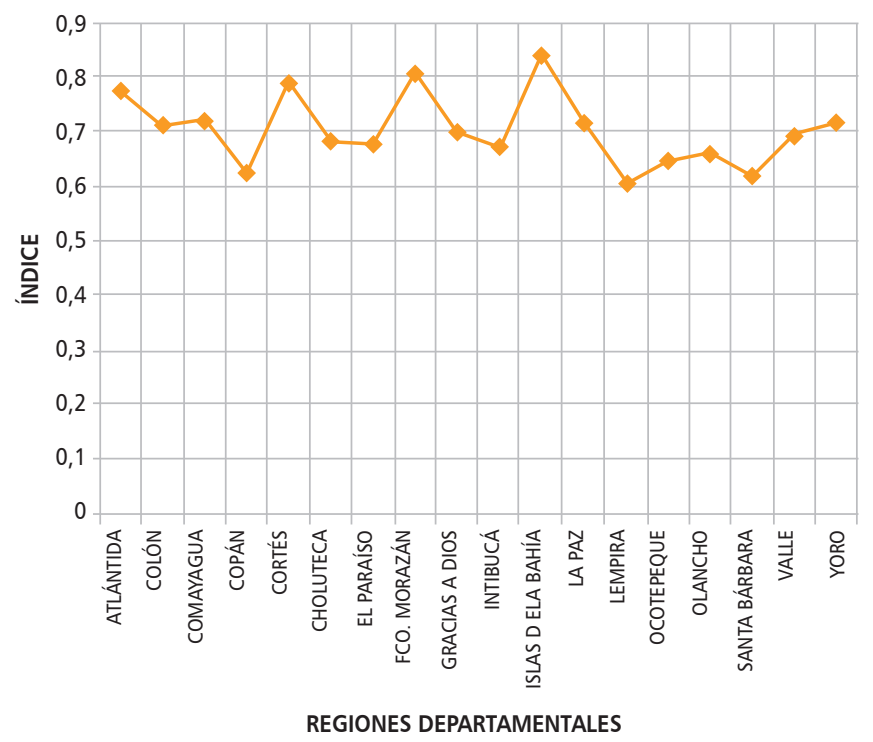

Fuente: Desarrollo Humano en Honduras limitado por la Inequidad.2009.PNUD.

3. El índice de educación que se hace mención en este documento se obtiene del dato de los valores del IDH y sus componentes para Honduras y sus departamentos en el 2009.

4. El índice de Ingreso al que hacemos referencia es una estimación de ingresos basada en los ingresos de los hogares provenientes por trabajo del respectivo departamento o municipio, y ajustada a los valores del PIB per cápita nacional en poder de paridad adquisitivo. El valor superior a 5 indica de extrema desigualdad en los grupos sociales (PNUD, 2009). 
Cuadro 3. Valores de índice de ingreso por departamento, año 2009.

\begin{tabular}{|l|c|}
\hline & DEPARTAMENTO \\
\hline Atlántida & .632 \\
\hline Colón & .576 \\
\hline Comayagua & .553 \\
\hline Copán & .531 \\
\hline Cortés & .668 \\
\hline Choluteca & .565 \\
\hline El Paraíso & .524 \\
\hline Francisco Morazán & .682 \\
\hline Gracias a Dios & .442 \\
\hline Intibucá & .451 \\
\hline Islas de la Bahía & .655 \\
\hline La Paz & .507 \\
\hline Lempira & .453 \\
\hline Ocotepeque & .523 \\
\hline Olancho & .554 \\
\hline Santa Bárbara & .515 \\
\hline Valle & .573 \\
\hline Yoro & .582 \\
\hline
\end{tabular}

Fuente: Desarrollo Humano en Honduras limitado por la Inequidad. 2009, PNUD.

\section{Gráfico 3. Índice de ingreso, 2009}

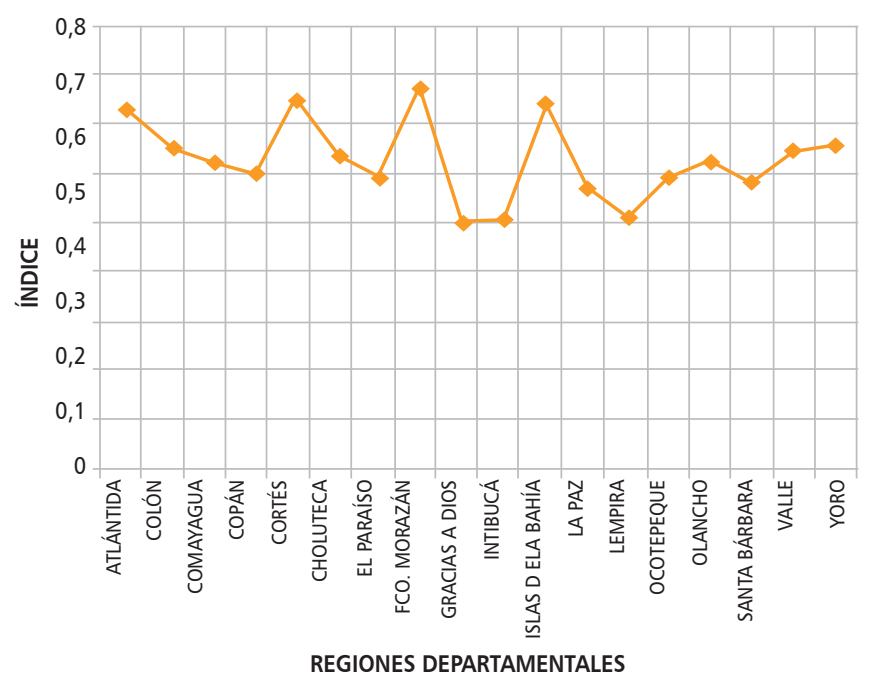

\section{Reflexión final}

La tasa de mortalidad infantil es un ejemplo de inequidad sanitaria. Estas inequidades son el resultado de desigualdades en el seno de las sociedades y entre sociedades. Las condiciones sociales y económicas y sus efectos en la vida de la población, determinan el riesgo de enfermar y las medidas que se adoptan para evitar que la población se enferme.

Dentro de los países, los datos muestran que la situación socioeconómica de una persona, incide en la salud. Existe un gradiente social de la salud que se traza desde la cúspide hasta la base del espectro socioeconómico. Se trata de un fenómeno mundial que se observa en los países de ingresos altos, medios y bajos. Este gradiente social de la salud implica que las inequidades sanitarias, afectan a todos (WHO, 2011).

Los datos muestran que la mayor mortalidad ocurre en los departamentos que tienen mejores índices de educación y de ingreso, como ser Francisco Morazán y Cortés. Es en estos departamentos en donde se encuentran las Regiones Metropolitanas que contienen los hospitales de referencia nacional que captan la mayor parte de los pacientes menores de 1 año que mueren. También se muestra que a nivel de educación, el gradiente entre el valor máximo y el mínimo es de 200 puntos (Francisco Morazán y Lempira).

En este sentido, la Organización Mundial de la Salud (OMS) establece que es necesario considerar condiciones de salud prioritaria y sus determinantes sociales, así como realizar exploraciones para lograr hacer frente a las desigualdades evitables e injustas en los niveles socioeconómicos de contexto, de exposición, de vulnerabilidad, así como los resultados en la salud y sus consecuencias sociales. El análisis debe ir mucho más allá de los conceptos para explorar como los determinantes sociales, como ser educación e ingreso, se pueden abordar en el mundo real (WHO, 2011).

Las desigualdades en salud, no se producen al azar. Por el contrario, el estado de salud guarda relación con las características biológicas y sociales de los individuos, la organización económica y política, la estructura social y el trasfondo cultural como también con los procesos demográficos y macroecológicos. La tasa de mortalidad infantil, es un buen indicador para resumir la infraes- 
tructura sanitaria de una población y las condiciones ambientales, socioculturales y económicas que pueden actuar negativamente en la salud de las personas (Flores \& Cerda, 2010).

Lo anterior motiva a realizar estudios encaminados a explicar los determinantes que se encuentran en la tasa de mortalidad infantil de las regiones departamentales con mejores índices de educación y de ingreso. El gradiente existente tanto a nivel de educación como de ingreso, no es muy amplio, por lo que es importante realizar discusiones sobre los determinantes de la salud y la equidad sanitaria y cómo los programas han abordado los retos que enfrentan durante su ejecución.

Estudios realizados en Colombia señalan, que sin tener en cuenta a los factores biológicos, la mortalidad infantil es explicada por factores socioeconómicos como: las condiciones propias de la ocupación de aquellas personas que sean económicamente activas al interior del hogar y sobre todo, del jefe del hogar, niveles de educación y en especial el de la madre, nutrición del niño y de la madre, niveles de fecundidad, condiciones y calidad de la vivienda, asistencia médica a la madre gestante y al niño/a después del nacimiento, niveles de ingreso del hogar, costumbres sociales, hábitos higiénicos y de preparación de alimentos, utilización adecuada de los programas y centros de asistencia de salud pública (Urdinola, 1993).

Otros autores, como Mosley y Chen en 1984, han realizado investigaciones relacionando los factores socioeconómicos con la mortalidad infantil y son los que están relacionados con factores maternales, como la edad de la madre, paridez, intervalo intergenésico, alimentación materna exclusiva, niveles de fecundidad y de fertilidad; también hacen relación con factores ambientales que se relacionan con la presencia de enfermedades infectocontagiosas, la presencia de deficiencias nutricionales tanto de la madre como del niño, accidentalidad y control de enfermedades que incluye medidas preventivas y curativas en el niño (Urdinola, 1993).

En Honduras, las diferencias entre las regiones departamentales, a nivel de los índices revisados, no son amplias, pero marcan diferencias sustanciales en cuanto a las tasas de mortalidad observadas. Esto significa que se debe realizar un abordaje para tratar de entender los determinantes de la salud con un valor fundamental en los programas de salud pública y trabajar no sólo a nivel de sectores sino en las condiciones de salud para lo que se requiere una visión que implique la técnica y el liderazgo en las políticas de salud, asociado a esfuerzos sostenibles. Los programas de salud pública deben aprender a afrontar los retos económicos y sociales en las que existan desigualdades. En este estudio, se han desagregado los datos para lograr establecer la importancia de comparar el fenómeno de muerte en el grupo poblacional de menores de un año a través de información epidemiológica, en los diferentes escenarios geopolíticos, relacionándola con los índices de educación y de ingreso. Mejorar la información sobre el número de infantes que mueren y sus determinantes, ayudarán a los líderes a decidir sobre el mejor curso de acción.

\section{Bibliografía}

- Ahmad, O., López, A., \& Inoue, M. (2000). Reevaluación de la disminución de la mortalidad infantil. Ginebra: Bulletin of the World Health Organization.

- Behm, H. (2011). Determinantes económicos y sociales de la mortalidad en América Latina. Buenos Aíres. CELADE.

- Delgado, I. (2003). Mortalidad en Menores de 5 años en Chile y Condiciones Socieconòmicas. Cohorte de nacidos en 1997 fallecidos antes de 5 años. Santiago de Chile: Universidad de Chile. Facultad de Medicina. Escuela de Salud Pùblica.

- Dirección de Planificación, E. y. (2009). Análisis de la mortalidad infantil según condicionantes sociales de la salud en la Provincia de Mendoza. Mendoza: Ministerio de Salud-Mendoza.

- FEREMA. (2010). Informe de Progreso Educativo. Honduras. Tegucigalpa.

- Flores, M., \& Cerda, J. (2010). Evolución de la Desigualdad en la Mortalidad Infantil. Santiago: Rev Chil Pediatr2010;81(3):215-220. 
- Honduras, S. d. (2008). Guía para la vigilancia de la mortalidad en el menor de 5 años en Honduras. Tegucigalpa.

- Medina, M., \& Cerda, J. (2010). Nivel de Educaciòn Parental y Mortalidad Infantil. Santiago de Chile: Pontificia Universidad Católica de Chile.

- OMS, C. d. (2007). Determinantes Estructurales de las Desigualdades de Salud. Ginebra.

- OMS, C. d. (2009). Subsanar las desigualdades en una generación. Ginebra.

- PNUD. (1990). Desarrollo Humano. Informe 1990.

- PNUD. (2008). ODM4. Reducir la mortalidad de los niños menores de 5 años. Tegucigalpa.
- PNUD. (2009). Desarrollo Humano en Honduras: limitado por la inequidad territorial. Tegucigalpa.

- Salud, D. d. (2009). Boletín Epidemoológico de la República de Honduras. Tegucigalpa.

- Urdinola, P. (1993). Determinantes socieconómicos de la mortalidad infantil en Colombia, 1993. Bogotà: Revista Colombiana de Estadìstica. Junio 2011.vol 34.No.1.pp 39-72.

- WHO, T. (2011). Social determinants approaches to public health: from concept to practice. Geneva. 